\title{
Comparative study of gamma-ray emission from molecular clouds and star-forming galaxies
}

\author{
Fang-Kun Peng ${ }^{1,2,3,4}$, Shao-Qiang Xi ${ }^{2,4}$, Xiang-Yu Wang ${ }^{2,4}$, Qi-Jun Zhi ${ }^{1}$, and Di Li ${ }^{5,6}$ \\ ${ }^{1}$ Guizhou Provincial Key Laboratory of Radio Astronomy and Data Processing, Guizhou Normal University, Guiyang 550001, \\ PR China \\ e-mail: pengfangkun@163.com \\ 2 School of Astronomy and Space Science, Nanjing University, Nanjing 210093, PR China \\ e-mail: xywang@nju.edu.cn \\ ${ }^{3}$ Guangxi Key Laboratory for Relativistic Astrophysics, Nanning 530004, PR China \\ ${ }^{4}$ Key laboratory of Modern Astronomy and Astrophysics, Nanjing University, Ministry of Education, Nanjing 210093, PR China \\ ${ }^{5}$ National Astronomical Observatories, Chinese Academy of Sciences, Beijing 100012, PR China \\ e-mail: dili@nao.cas.cn \\ ${ }^{6}$ CAS Key Laboratory of FAST, NAOC, Chinese Academy of Sciences, Beijing 100101, PR China
}

Received 15 July 2018 / Accepted 6 November 2018

\begin{abstract}
Star-forming regions on different scales, such as giant molecular clouds in our Galaxy and star-forming galaxies, emit GeV gammarays. These are thought to originate from hadronic interactions of cosmic-ray (CR) nuclei with the interstellar medium. It has recently been shown that the gamma-ray luminosity $\left(L_{\gamma}\right)$ of star-forming galaxies is well correlated with their star formation rates (SFR). We investigated Fermi data of eight Galactic molecular clouds in the Gould belt and found that molecular clouds do not follow the $L_{\gamma}$ - SFR correlation of star-forming galaxies. We also compared the scaling relations of gamma-ray luminosity, SFR, and the gas mass for molecular clouds and star-forming galaxies. Using a multiple-variable regression analysis, we found different dependences of gamma-ray emission on SFR or mass for molecular clouds and star-forming galaxies. This suggests that different mechanisms may govern the production of gamma-rays in these two types of sources. Specifically, the strong dependence on mass supports that gammaray emission of molecular clouds primarily comes from passive interaction by diffuse Galactic CRs, whereas the strong dependence on SFR supports that gamma-ray emission of star-forming galaxies originates from CRs that are accelerated by local active sources.
\end{abstract}

Key words. cosmic rays - ISM: clouds - gamma rays: ISM - methods: statistical

\section{Introduction}

Several nearby star-forming and starburst galaxies have been identified to be $\mathrm{GeV}$ to $\mathrm{TeV}$ gamma-ray sources (e.g., Acero et al. 2009; VERITAS Collaboration 2009; Abdo et al. 2010a; Ackermann et al. 2012a; Tang et al. 2014; Peng et al. 2016; Griffin et al. 2016; Ackermann et al. 2017). Cosmic rays (CRs) accelerated by supernova remnants (SNRs) or the stellar wind of massive stars interact with the interstellar medium (ISM) and produce neutron pions (schematically written as $p+p \rightarrow$ $p+\pi^{0}+$ other products), which in turn decay into high-energy gamma-rays $\left(\pi^{0} \rightarrow \gamma+\gamma\right)$. Interestingly, with the early Fermi Large Area Telescope (LAT) data, Abdo et al. (2010a) found a correlation between the gamma-ray $(>100 \mathrm{MeV})$ luminosity $\left(L_{\gamma}\right)$ and star formation rate (SFR) for nearby star-forming galaxies. Based on three years of Fermi-LAT data, a tight correlation between the gamma-ray luminosity and total infrared luminosity $(8-1000 \mu \mathrm{m})$ over $4-5$ orders of magnitudes has been reported for star-forming galaxies by Ackermann et al. (2012a). Since the total infrared luminosity is an indicator of the SFR of star-forming galaxies and galaxies that are not yet detected in gamma-rays are also taken into account to reduce sample selection effects, this then suggests that the positive correlation between the gamma-ray luminosity and SFR $\left(L_{\gamma}-\mathrm{SFR}\right)$ is robust. Lately, this correlation has been extended to ultraluminous infrared galaxies, with the detection of gamma-ray emission from Arp 220 (Peng et al. 2016; Griffin et al. 2016). This strengthens the connection between star formation process and gamma-ray emission on a larger luminosity scale.

Molecular clouds, which are the sites of star formation in our Galaxy, are also sources of gamma-ray emission (e.g., Ackermann et al. 2012b,c,d). The widely accepted explanation of the gamma-ray emission of molecular clouds is that the clouds are passive targets for interaction with diffuse Galactic CRs (e.g., Aharonian 2001; Grenier et al. 2005; Gabici et al. 2007). The passive-target scenario assumes that Galactic cosmic rays can freely penetrate the clouds and enter into the core region. This scenario has gained support from the fairly uniform distribution of gamma-ray emissivity per gas nucleon in the Gould Belt clouds and in the Local Arm (Abdo et al. 2009, 2010b; Ackermann et al. 2011a, 2012c,d; Casandjian 2012).

On the other hand, there have been suggestions that molecular clouds contain active sources of CRs. Embedded young stellar objects (YSOs) in giant molecular clouds are proposed to be sources of high-energy cosmic rays (e.g., Araudo et al. 2007; Bosch-Ramon et al. 2010; Maurin et al. 2016). The strong stellar wind activity in these objects generates large bubbles and induces collective effects that could accelerate particles up to high energy and produce gamma-rays (e.g., Ackermann et al. 2011b; Yang \& Aharonian 2017; Yang et al. 2018; Aharonian et al. 2018). For low-mass YSOs such as T Tauri protostars, their jets and winds should be unimportant. However, these 
objects have bubbles and outflows that seem to sustain turbulence ( $\mathrm{Li}$ et al. 2015). This would mean that particles could also be accelerated to relativistic energies by turbulence or a magnetic reconnection process (del Valle et al. 2011).

It has been clear that stars are born in the molecular gas, especially in regions with dense molecular gas, rather than those primarily with atomic hydrogen. The dense region within molecular clouds collapses and then forms new stars. The SFR of molecular clouds estimated from the far-infrared emission correlates well with the dense molecular gas mass traced by $\mathrm{HCN}$ for galaxies (e.g., Gao \& Solomon 2004). This correlation continues to dense Galactic cores over a large scale of magnitude in SFR (e.g., Wu et al. 2005; Lada et al. 2010). As molecular clouds are a scaled down version of star-forming galaxies in some sense, the question arises whether the $L_{\gamma}-$ SFR correlation can extend to the scale of Galactic molecular clouds.

To study whether the SFR plays an important role in producing gamma-ray emission in molecular clouds, we here conduct a comparative study of the correlation between gamma-ray luminosity and SFR for Galactic molecular clouds and star-forming galaxies. Furthermore, we systematically investigate the correlations among $L_{\gamma}$, SFR, and gas mass $M$ to study the relative dependence of $L_{\gamma}$ on SFR and gas mass. We analyzed the FermiLAT data of eight Galactic molecular clouds in the Gould Belt. We noted that the Fermi-LAT data of local molecular clouds of the Gould Belt had been used to probe the CR properties by several groups (Neronov et al. 2012, 2017; Yang et al. 2014; Shen et al. 2018). However, these works focused on deriving the spectrum of the parent CRs through the resolved gamma-ray spectral analysis and compared it with the measurements of local Galactic CRs. Their relatively large offsets from the Galactic plane ensure little contamination from the diffuse Galactic gamma-ray emission. The close distances lead to a high detection significance of the gamma-ray emission. These make them good candidates for our study. The structure of the paper is as follows. In Sect. 2, we describe the data reduction and results of the Fermi-LAT observations. In Sect. 3, we study whether the $L_{\gamma}-\mathrm{SFR}$ correlation of galaxies can extend to the scale of Galactic molecular clouds. In Sect. 4, we present the study on the correlation among gamma-ray luminosity $L_{\gamma}$, SFR, and the gas mass $M$. In Sect. 5, we discuss the implications of these two-parameter and three-parameter correlations. Finally, we conclude in Sect. 6.

\section{Fermi-LAT data reduction}

The LAT on board the Fermi mission is a pair-conversion instrument that is sensitive to $\mathrm{GeV}$ emission (Atwood et al. 2009). We accumulated data events from the start (MET 239557417) to 2017 April 6 (MET 513164606) to study gamma-ray emission from the Galactic molecular clouds. The basic information of the clouds, including the position, size, mass, and distance, is listed in Tables 1 and 2. The current Fermi-LAT Pass 8 SOURCE data (P8R2 Version 6) and the standard Fermi science tools version v10r0p5 were used. All FRONT+BACK converting photons with energies higher than $0.3 \mathrm{GeV}$ were taken into consideration to reduce the contamination from poor angular resolution events at lower energies. To limit the gamma-rays produced by $\mathrm{CR}$ interactions in the upper atmosphere, the maximum zenith-angle cut $z_{\max }=90^{\circ}$ was required. The expression of (DATA_QUAL $>0$ ) \& \& (LAT_CONFIG ==1) was used to further filter the data in the gtmktime. We performed the binned maximum likelihood analysis on a region of interest (ROI) with a radius of $10^{\circ}$ centered on the position of each
Table 1. Basic information on Galactic molecular clouds.

\begin{tabular}{lcccc}
\hline \hline Name & $\begin{array}{c}\text { Distance } \\
(\mathrm{pc})\end{array}$ & $M_{\text {total }, \odot}$ & $M_{\text {dense, } \odot}$ & $\begin{array}{c}\text { SFR } \\
\left(10^{-6} M_{\odot} \mathrm{yr}^{-1}\right)\end{array}$ \\
\hline RCrA & $148 \pm 30^{a}$ & 1137 & 258 & 25 \\
Oph & $119 \pm 6^{b}$ & 14165 & 1296 & 79 \\
Perseus & $240 \pm 13^{c}$ & 18438 & 1880 & 150 \\
Taurus & $153 \pm 8^{c}$ & 14964 & 1766 & 84 \\
Orion A & $371 \pm 10^{d}$ & 67714 & 13721 & 715 \\
Orion B & $398 \pm 12^{d}$ & 71828 & 7261 & 159 \\
Chamaeleon & $200^{e}$ & $5000^{e}$ & $342^{f}$ & $29^{g}$ \\
Mon R2 & $830^{h}$ & $40000^{h}$ & $2031^{h}$ & $82^{i}$ \\
\hline
\end{tabular}

Notes. The third and forth columns are the total masses and dense masses estimated from the infrared extinction map at $A_{K} \geq 0.1 \mathrm{mag}$ and $A_{K} \geq 0.8 \mathrm{mag}$, respectively (Lada et al. 2010). The last column is the SFR derived from the YSO observation. The masses and SFRs not marked are from Lada et al. (2010).

References. ${ }^{(a)}$ Knude (2010); ${ }^{(b)}$ Lombardi et al. (2008); ${ }^{(c)}$ Lombardi et al. (2010); ${ }^{(d)}$ Lombardi et al. (2011); ${ }^{(e)}$ Luhman (2008); ${ }^{(f)}$ Mizuno et al. (1999); ${ }^{(g)}$ Heiderman et al. (2010); ${ }^{(h)}$ Carpenter \& Hodapp (2008); ${ }^{(i)}$ Pokhrel et al. (2016).

Galactic molecular cloud. We used the 3FGL (Acero et al. 2015) to generate the source model containing the position and spectral definition for all the point sources and diffuse emission within $15^{\circ}$ of the ROI center. The extragalactic diffuse model iso_P8R2_SOURCE_V6_v06.txt was also included.

All molecular clouds in our sample extend several degrees above the Galactic plane on the sky. A comparison of the gamma-ray counts map with $\mathrm{CO}$ intensity reveals a good correlation between the gamma-ray and $\mathrm{CO}$ emission. The $\mathrm{CO}$ distribution in the direction of sight was reduced to a single peak by integrating the $\mathrm{CO}$ cube in the spatial dimension (Dame et al. 2001). We particularly focused on the integral gamma-ray flux of each molecular cloud for the correlation studies. Because the Galactic diffuse gamma-ray emission in the ROI is dominated by the interaction between CRs and molecular clouds and neutral hydrogen is distributed diffusively in our Galaxy, we adopted the following method. The templates for modeling the spatial and spectral distribution of molecular clouds were extracted from the standard Galactic diffuse emission model, that is, a cube file named as gll_iem_v06.fits, provided by the Fermi-LAT Collaboration. We selected one region nearby with the same size for each molecular cloud as the background without significant $\mathrm{CO}$ emission. In each energy band, we obtained the average value in the background region, which was also used to model the residual Galactic diffuse emission of the molecular cloud. Then we obtained the molecular cloud templates by subtracting the above value from the standard gll_iem_v06.fits. After the source model was created, the standard commands gtbin, gtltcube, gtexpcube2, and gtsrcmaps were successively executed, then a maximum likelihood analysis was performed in binned mode using the tool gtlike ${ }^{1}$.

Cosmic rays generate diffuse gamma-ray emission by interacting with interstellar gas and magnetic fields during their propagation through the Galaxy. One way to derive the spatial and spectral information of the diffuse gamma-ray emission templates is to use the GALPROP code $^{2}$. We therefore checked

\footnotetext{
1 https://fermi.gsfc.nasa.gov/ssc/data/analysis/ scitools/binned_likelihood_tutorial.html

2 https://galprop.stanford.edu/
} 
Table 2. Parameters and gamma-ray fluxes of Galactic molecular clouds.

\begin{tabular}{lccccccc}
\hline \hline Name & $\left(l_{s}\left(^{\circ}\right), b_{s}\left(^{\circ}\right)\right)$ & $\theta\left(^{\circ}\right)$ & $\left(l_{b}\left({ }^{\circ}\right), b_{b}\left({ }^{\circ}\right)\right)$ & Flux $_{0.1-100 \mathrm{GeV}}$ & Error & Flux $_{1-500 \mathrm{GeV}}$ & Error \\
\hline RCrA & $(0.56,-19.63)$ & 3 & $(6.94,-19.63)$ & 0.91 & 2.24 & 0.87 & 0.15 \\
Oph & $(355.81,16.63)$ & 5 & $(34.94,16.63)$ & 11.3 & 0.56 & 12.1 & 2.80 \\
Perseus & $(159.31,-20.25)$ & 4 & $(148.44,-19.88)$ & 4.72 & 0.29 & 4.82 & 0.76 \\
Taurus & $(173.19,-14.75)$ & 6 & $(143.94,-19.50)$ & 16.4 & 6.69 & 15.4 & 1.27 \\
Orion A & $(212.19,-19.13)$ & 4 & $(233.69,-19.13)$ & 9.35 & 4.06 & 9.10 & 9.34 \\
Orion B & $(204.56,-13.75)$ & 4 & $(233.69,-19.13)$ & 9.26 & 10.2 & 8.70 & 14.3 \\
Chamaeleon & $(300.43,-16.13)$ & 5.5 & $(283.81,-16.13)$ & 4.56 & 1.21 & 4.46 & 3.53 \\
Mon R2 & $(213.81,-12.63)$ & 1.5 & $(233.81,-18.75)$ & 1.91 & 1.17 & 1.81 & 9.47 \\
\hline RCrA & $(0.56,-19.63)$ & 3 & $(6.94,-19.63)$ & 0.73 & 1.80 & 0.33 & 0.06 \\
Oph & $(355.81,16.63)$ & 5 & $(34.94,16.63)$ & 10.2 & 0.51 & 5.46 & 1.27 \\
Perseus & $(159.31,-20.25)$ & 4 & $(148.44,-19.88)$ & 4.02 & 0.24 & 2.01 & 0.32 \\
Taurus & $(173.19,-14.75)$ & 6 & $(143.94,-19.50)$ & 13.6 & 5.56 & 6.32 & 0.52 \\
Orion A & $(212.19,-19.13)$ & 4 & $(233.69,-19.13)$ & 7.75 & 3.37 & 3.73 & 3.83 \\
Orion B & $(204.56,-13.75)$ & 4 & $(233.69,-19.13)$ & 7.56 & 8.32 & 3.55 & 5.83 \\
Chamaeleon & $(300.43,-16.13)$ & 5.5 & $(283.81,-16.13)$ & 3.78 & 1.00 & 1.86 & 1.47 \\
Mon R2 & $(213.81,-12.63)$ & 1.5 & $(233.81,-18.75)$ & 1.56 & 0.95 & 0.75 & 3.93 \\
\hline
\end{tabular}

Notes. The second and third columns are the position and size of the source region. The forth column is the position of the background estimation region, whose size is the same as the source region. The fluxes and errors above the double horizontal lines in $0.1-100 \mathrm{GeV}$ are in units of $10^{-7} \mathrm{ph} \mathrm{cm}^{-2} \mathrm{~s}^{-1}$ and $10^{-9} \mathrm{ph} \mathrm{cm}^{-2} \mathrm{~s}^{-1}$, respectively. The fluxes and errors above the double horizontal lines in $1-500 \mathrm{GeV}$ are in units of $10^{-8} \mathrm{ph} \mathrm{cm}^{-2} \mathrm{~s}^{-1}$ and $10^{-10} \mathrm{ph} \mathrm{cm}^{-2} \mathrm{~s}^{-1}$, respectively. The fluxes and errors below the double horizontal lines are in units of $10^{-10} \mathrm{erg} \mathrm{cm}^{-2} \mathrm{~s}^{-1}$ and $10^{-12} \mathrm{erg} \mathrm{cm}^{-2} \mathrm{~s}^{-1}$, respectively.

Table 3. Change in gamma-ray flux of the first three molecular clouds for different background models.

\begin{tabular}{lccccccccccccccccc}
\hline \hline Name & 1 & 2 & 3 & 4 & 5 & 6 & 7 & 8 & 9 & 10 & 11 & 12 & 13 & 14 & 15 & 16 \\
\hline RCrA & 0.13 & 0.06 & 0.15 & 0.07 & 0.11 & 0.03 & 0.14 & 0.04 & 0.11 & 0.03 & 0.13 & 0.04 & 0.09 & -0.01 & 0.12 & 0.01 \\
Oph & 0.05 & 0.05 & 0.05 & 0.06 & 0.05 & 0.06 & 0.06 & 0.07 & 0.05 & 0.06 & 0.06 & 0.07 & 0.05 & 0.08 & 0.07 & 0.08 \\
Perseus & 0.07 & 0.02 & 0.08 & 0.05 & 0.07 & 0.06 & 0.09 & 0.05 & 0.06 & 0.02 & 0.08 & 0.04 & 0.07 & 0.02 & 0.08 & 0.05 \\
\hline
\end{tabular}

Notes. The values represent the ratio between $(\mathrm{F} 2-\mathrm{F} 1)$ and $\mathrm{F} 1$, where $\mathrm{F} 1$ is derived using the method in the second paragraph of Sect. 2, and F2 is derived using the method in the third paragraph of Sect. 2. 1: Lorimer, $4 \mathrm{kpc}, 150 \mathrm{~K}$, and $2 \mathrm{mag}$; 2: Lorimer, $4 \mathrm{kpc}, 150 \mathrm{~K}$, and $5 \mathrm{mag}$; 3: Lorimer, $4 \mathrm{kpc}, 10^{5} \mathrm{~K}$, and $2 \mathrm{mag}$; 4: Lorimer, $4 \mathrm{kpc}, 10^{5} \mathrm{~K}$, and $5 \mathrm{mag}$; 5: Lorimer, $10 \mathrm{kpc}, 150 \mathrm{~K}$, and $2 \mathrm{mag}$; 6: Lorimer, $10 \mathrm{kpc}, 150 \mathrm{~K}$, and $5 \mathrm{mag}$; 7: Lorimer, $10 \mathrm{kpc}, 10^{5} \mathrm{~K}$, and $2 \mathrm{mag}$; 8: Lorimer, $10 \mathrm{kpc}, 10^{5} \mathrm{~K}$, and $5 \mathrm{mag}$; 9: SNR, $4 \mathrm{kpc}, 150 \mathrm{~K}$, and $2 \mathrm{mag}$; 10 : SNR, $4 \mathrm{kpc}, 150 \mathrm{~K}$, and $5 \mathrm{mag}$; 11: SNR, $4 \mathrm{kpc}, 10^{5} \mathrm{~K}$, and $2 \mathrm{mag}$; 12: SNR, $4 \mathrm{kpc}, 10^{5} \mathrm{~K}$, and $5 \mathrm{mag}$; 13 : SNR, $10 \mathrm{kpc}, 150 \mathrm{~K}$, and $2 \mathrm{mag}$; 14 : SNR, $10 \mathrm{kpc}, 150 \mathrm{~K}$, and $5 \mathrm{mag}$; 15: SNR, $10 \mathrm{kpc}, 10^{5} \mathrm{~K}$, and $2 \mathrm{mag}$; 16: SNR, $10 \mathrm{kpc}, 10^{5} \mathrm{~K}$, and 5 mag.

the results on a diffuse emission model that did not include a gas component with the help of GALPROP. We used models for the predicted Galactic diffuse gamma-ray emission obtained from the Fermi-LAT collaboration work (Ackermann et al. 2012d), which provides 128 sets of maps corresponding to different model parameters. We tested 16 of these 128 templates and chose two each for the CR source distribution (Lorimer pulsars; Lorimer et al. 2006), SNRs (Case \& Bhattacharya 1998), vertical boundaries (4 and $10 \mathrm{kpc}$ ), spin temperature for the optical depth correction ( 150 and $\left.10^{5} \mathrm{~K}\right)$, and $E(B-V)$ magnitude cut (2 and $5 \mathrm{mag}$ ). We did not include the $\mathrm{H}_{2}$ component in these Galprop simulations. We used the spatial templates for pion-decay, bremsstrahlung radiation, and inverse Compton gamma-rays generated by GALPROP ${ }^{3}$ (Vladimirov et al. 2011) to replace the background model. The gamma-ray emissions of molecular clouds using the above 16 background models are consistent with those in Table 2, and the fluxes change at most by $\sim 15 \%$ in these models; see Table 3 . These small differences do not affect our statistical results and conclusion.

\footnotetext{
3 https://galprop.stanford.edu/webrun.php
}

The integral gamma-ray fluxes of molecular clouds are presented in Table 2. To conform with the energy range of the data for the star-forming galaxies, we extrapolated the flux in $0.3-100 \mathrm{GeV}$ to that in the energy range of $0.1-100 \mathrm{GeV}$. The statistical error is small due to the high-significance detection. The data of gamma-ray emission in $0.1-100 \mathrm{GeV}$ from star-forming galaxies were taken from previous publications (Ackermann et al. 2012a; Tang et al. 2014; Peng et al. 2016).

In order to reduce the possible contribution from leptonic emission, such as inverse Compton scatter and bremsstrahlung radiation at low energies, which would overestimate the flux due to hadronic CRs interaction, we also considered the Fermi-LAT data in $1-500 \mathrm{GeV}$. Moreover, the possible gamma-ray contamination emission from unresolved sources such as pulsars was also suppressed by increasing the threshold energy for the data analysis (Abdo et al. 2013). To obtain the gamma-ray luminosity in $1-500 \mathrm{GeV}\left(L_{1-500 \mathrm{GeV}}\right)$, we performed a likelihood analysis of the latest Fermi-LAT data following a method that was similar to the previously used method. The results are shown in Tables 2 and 4 . 

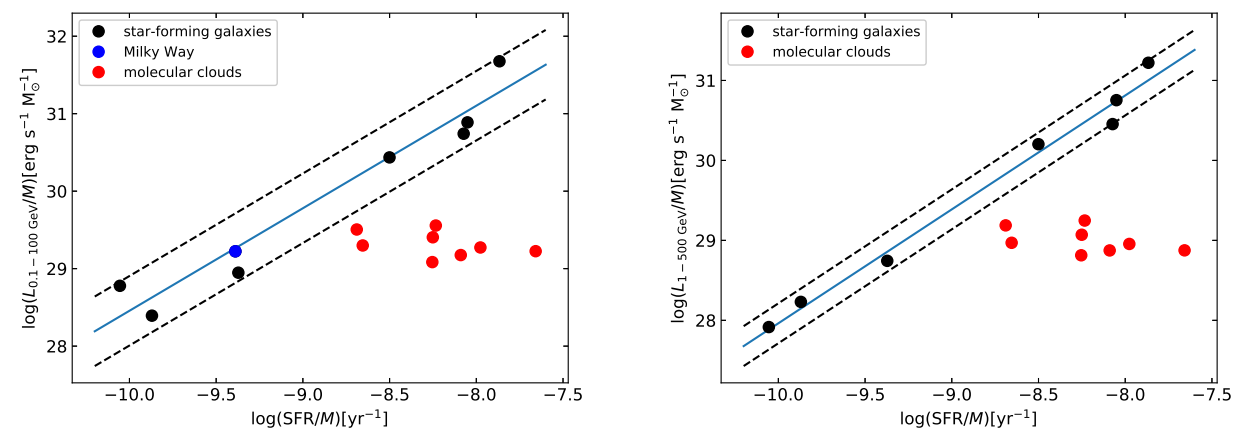

Fig. 1. Relation between gamma-ray emissivity (left panel: $0.1-100 \mathrm{GeV}$; right panel: $1-500 \mathrm{GeV}$ ) and SFR per unit mass for molecular clouds and star-forming galaxies. The best-fit lines together with their $1 \sigma$ dispersion regions are shown with solid and dashed lines, respectively.

\section{Do molecular clouds follow the $L_{\gamma}-$ SFR correlation of star-forming galaxies?}

As molecular clouds are a scaled-down version of star-forming galaxies in some sense, we first studied whether the $L_{\gamma}-$ SFR correlation of galaxies can extend to the scale of Galactic molecular clouds. To do this, we checked whether the Galactic molecular clouds fall onto the correlation line in the $L_{\gamma}-\mathrm{SFR}$ diagram of star-forming galaxies. Since $L_{\gamma}$ and SFR of molecular clouds and star-forming galaxies span 7-8 orders of magnitude, we reduced the dynamic range from clouds to starforming galaxies by dividing the gamma-ray luminosity and SFR by the gas mass $M$. We compared the gamma-ray emissivity $\left(L_{\gamma} / M\right)$ and SFR per unit mass for molecular clouds and star-forming galaxies. Although the methods for determining the gas masses for molecular clouds and star-forming galaxies are different, it has been proved that the molecular-linederived masses and the extinction-derived masses accurately reflect the same material (Lada et al. 2012). We computed the average integral $>100 \mathrm{MeV}$ gamma-ray emissivity per hydrogen atom of molecular clouds using the following form: $q_{\gamma}=$ $8.0 \times 10^{-27} \frac{F_{\gamma}}{10^{-7} \mathrm{ph} \mathrm{cm}^{-2} \mathrm{~s}^{-1}}\left(\frac{d}{1 \mathrm{kpc}}\right)^{2}\left(\frac{M_{\mathrm{gas}}}{10^{5} M_{\odot}}\right)^{-1}$, where the emissivity $q_{\gamma}$ is in unit of $\mathrm{ph} \mathrm{s}^{-1} \mathrm{sr}^{-1} \mathrm{H}^{-1}, F_{\gamma}$ is the integral photon flux, $M_{\text {gas }}$ is the total gas content of the molecular cloud, and $d$ is the distance to Earth. The mean value of the ratio between the measured integral gamma-ray emissivity of local atomic hydrogen (Abdo et al. 2009) and that of the sample molecular clouds in our work is $1.13 \pm 0.69$, and the median value is 0.90 , indicating that the gamma-ray emissivities of molecular clouds are quite close to the emissivity of local atom hydrogen.

The results are reported in Fig. 1. A correlation is evident between the gamma-ray emissivity $\left(L_{\gamma} / M\right)$ and SFR per unit mass for star-forming galaxies, but the molecular clouds significantly deviate from this correlation. The Pearson correlation coefficient of star-forming galaxies is $r>0.9$ and the chance probability is $p<10^{-4}$. The derived total dispersions, including the intrinsic scatter of the data set and the statistic scatter, are 0.45 and 0.24 for the gamma-ray emissivity $\left(L_{\gamma} / M\right)$ in $0.1-100 \mathrm{GeV}$ and $1-500 \mathrm{GeV}$, respectively. The gamma-ray emissivities $\left(L_{\gamma} / M\right)$ of molecular clouds are distributed in a narrow space and are comparable to the mean value of the Milky Way, which contains a great variety of different molecular clouds with different star-forming activities. The different scalings imply that the gamma-ray emission of molecular clouds and star-forming galaxies has a different origin. The roughly constant gamma-ray emissivity among the clouds as well as the Milky Way supports the hypothesis that the gamma-ray emission of clouds is due to passive interactions by the diffuse Galactic CRs. This wide range of SFR/M may reflect the variations in the fractions of dense gas. The physical interpretation behind it could be due to the cloud evolution. The evolution of molecular clouds is controlled by a complex interplay of large-scale phenomena and microphysics, such as turbulence, magnetic field, outflow of young stellar objects, far-ultraviolet radiation, CR radiation, gas, and dust (e.g., Vázquez-Semadeni et al. 2011, and reference therein).

\section{Multiple-variable regression analysis}

In this section, we conduct a multiple-variable regression analysis to study the correlations among the parameters of gamma-ray luminosity, SFR, and $M$ for molecular clouds and star-forming galaxies, respectively. The underlying theory for this statistical analysis is that gamma-ray emission should depend on both the sources of CRs and the target gases. The sources of Galactic CRs could be SNRs and/or young stellar objects, which are indicated by the SFR. We therefore studied the correlation between gamma-ray luminosity and SFRs for molecular clouds and starforming galaxies. Furthermore, we systematically investigated the correlations among gamma-ray luminosity, SFR, and gas mass $M$ to study the relative dependence of gamma-ray emission on SFR and gas mass. Through a comparative and statistic analysis, we examine whether the SFR plays an important role in producing gamma-ray emission in molecular clouds, and we study the difference of gamma-ray emission processes in molecular clouds and star-forming galaxies.

Generally speaking, the results of the regression analysis depend on the choice of dependent and independent variables (Isobe et al. 1990; Feigelson \& Babu 1992), especially in our case, where the sample size is small and the intrinsic data scatter is large. The bisector or orthogonal method could be adopted to solve the problem from the point of view of mathematics. However, from a physical point of view, the independent and dependent variables are believed to be clear. For the given data set, SFR stands for the sources of CRs, the gas mass $M$ stands for the target material, and $L_{\gamma}$ is the result of CR interaction between sources and target. Therefore, $L_{\gamma}$ was chosen as the independent variable in the following analysis.

The two-parameter correlation between $L_{\gamma}$ and SFR for nearby star-forming galaxies has been found for the first time by Abdo et al. (2010a), and it has been confirmed by follow-up studies (Ackermann et al. 2012a; Tang et al. 2014; Peng et al. 2016; Griffin et al. 2016). This discovery is linked to the relation between CRs and SFR, although its origin is not yet fully understood. Here we explore the two-parameter correlations between gamma-ray luminosity and SFR (or gas mass) for star-forming galaxies and clouds to determine the roles that the SFR plays in producing gamma-ray emission and to understand the physical nature behind the scaling relations. 


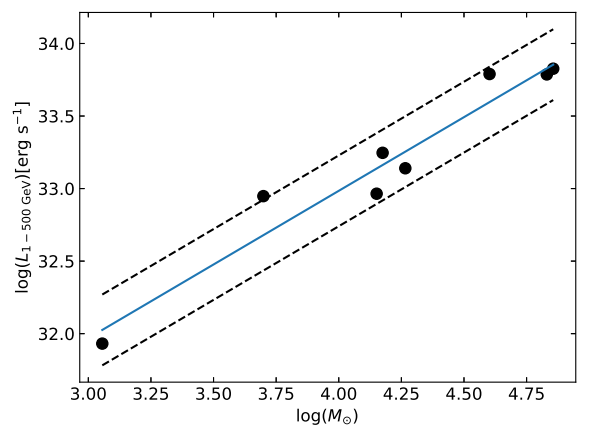

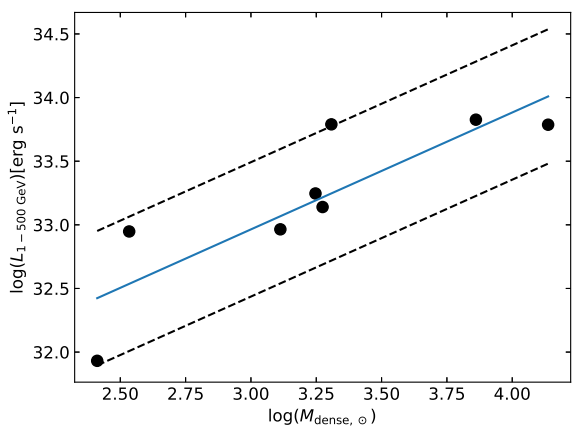

Fig. 2. Two-parameter correlation between $L_{1-500 \mathrm{GeV}}$ and total gas mass (left panel) and dense gas mass (right panel) for molecular clouds. The best-fit lines together with their $1 \sigma$ dispersion regions are shown with solid and dashed lines, respectively.

Table 4. Parameters and gamma-ray luminosities of star-forming galaxies.

\begin{tabular}{lccccc}
\hline \hline Name & $\begin{array}{c}\text { Distance } \\
(\mathrm{Mpc})\end{array}$ & $\begin{array}{c}L_{0.1-100 \mathrm{GeV}} \\
\left(\mathrm{erg} \mathrm{s}^{-1}\right)\end{array}$ & $\begin{array}{c}L_{1-500 \mathrm{GeV}} \\
\left(\mathrm{erg} \mathrm{s}^{-1}\right)\end{array}$ & $\begin{array}{c}\mathrm{SFR} \\
\left(M_{\odot} \mathrm{yr}^{-1}\right)\end{array}$ & $M_{\odot, 9}$ \\
\hline Milky Way & $\ldots$ & $(8.20 \pm 2.40) \times 10^{38}$ & $\ldots$ & $1-3^{a}$ & $4.90 \pm 0.45^{b}$ \\
LMC & 0.05 & $(4.70 \pm 0.50) \times 10^{37}$ & $(2.94 \pm 0.05) \times 10^{37}$ & $0.20-0.25^{c}$ & $0.53 \pm 0.02^{d}$ \\
SMC & 0.06 & $(1.10 \pm 0.30) \times 10^{37}$ & $(7.57 \pm 0.34) \times 10^{36}$ & $0.04-0.08^{e}$ & $0.45 \pm 0.04^{f}$ \\
M31 & 0.78 & $(4.60 \pm 1.00) \times 10^{38}$ & $(6.31 \pm 0.21) \times 10^{37}$ & $0.35-1^{a}$ & $7.66 \pm 2.1^{g}$ \\
NGC 253 & 2.5 & $(6.00 \pm 2.00) \times 10^{39}$ & $(3.51 \pm 0.40) \times 10^{39}$ & $3.5-10.4^{h}$ & $2.20^{i}$ \\
M82 & 3.4 & $(1.50 \pm 0.30) \times 10^{40}$ & $(7.74 \pm 0.28) \times 10^{39}$ & $13-33^{j}$ & $2.72^{i}$ \\
NGC 2146 & 15.2 & $(4.62 \pm 2.43) \times 10^{40}$ & $(3.39 \pm 1.20) \times 10^{40}$ & $26.6-79.7^{k}$ & $5.98^{i}$ \\
Arp 220 & 74.7 & $(1.78 \pm 0.30) \times 10^{42}$ & $(6.24 \pm 2.21) \times 10^{41}$ & $254.8-764.3^{k}$ & $37.5^{i}$ \\
\hline
\end{tabular}

Notes. The distances are provided by Ackermann et al. (2012a). The gamma-ray luminosities of the Milky Way $L_{0.1-100 \mathrm{GeV}}$ have been estimated using a numerical model of CR propagation and interactions in the ISM (Strong et al. 2010). $M_{\odot, 9}$ is $M_{\odot} / 10^{9}$.

References. ${ }^{(a)}$ Yin et al. (2009); ${ }^{(b)}$ Paladini et al. (2007); ${ }^{\left({ }^{c}\right.}$ Hughes et al. (2007); ${ }^{(d)}$ Staveley-Smith et al. (2003); Fukui et al. (2008); ${ }^{\left({ }^{e}\right)}$ Wilke et al. (2004); ${ }^{(f)}$ Stanimirovic et al. (1999); Leroy et al. (2007); ${ }^{(g)}$ Braun et al. (2009); Nieten et al. (2006); ${ }^{(h)}$ Lenc \& Tingay (2006); ${ }^{(i)}$ Gao \& Solomon (2004); ${ }^{(j)}$ Förster Schreiber et al. (2003); ${ }^{(k)}$ Cillis et al. (2005).

We modeled the two-parameter correlation using the form $z=a+b \times x$. To obtain the best-fitting parameters to the observational data with the two-parameter correlation analysis, we used the maximum likelihood approach. The joint likelihood function for two-parameter analysis is

$\mathcal{L}(a, b, \sigma)=\prod_{i} \frac{1}{\sqrt{2 \pi \sigma^{2}}} \times e^{-\frac{\left(z_{i}-a-b \times x_{i}\right)^{2}}{2 \sigma^{2}}}$,

where $i$ is the corresponding serial number of molecular clouds or star-forming galaxies in our sample, $z$ is $L_{\gamma}$, and $x$ is SFR (or $M$ ). They all are derived in logarithmic space. Since no error bars for SFR and $M$ are available, and very small statistic errors in $L_{\gamma}$ for some molecular clouds (see Table 2) would lead to relatively large weights, no measurement errors were considered. $\sigma$ is introduced to accommodate intrinsic scatter and measurement errors. The coefficients of $a, b$, and $\sigma$ are constrained simultaneously by maximizing the joint likelihood function.

For each sample of molecular clouds and star-forming galaxies, we used the Python Markov chain Monte Carlo module EMCEE (Foreman-Mackey et al. 2013) to explore the posterior distributions of parameters of $a, b$, and $\sigma$. We derived the dispersion $(\delta)$ of a regression model with standard deviation of $z^{r}$ from $z$, where $r$ marks the $z$ value derived from the regression model.

We applied this two-parameter correlation analysis to molecular clouds and star-forming galaxies using the forms $\log L_{\gamma}=$ $a+b \log (\mathrm{SFR})$ or $\log L_{\gamma}=a+b \log (M)$. The results of the correlations are shown in Tables 5 and 6 . The results in Tables 5 and 6 show at first glance that all correlations between $L_{1-500 \mathrm{GeV}}$ and SFR or $M$ are significant from the statistical point of view, even
Table 5. Results of the two-parameter correlation for $L_{1-500 \mathrm{GeV}}$ and SFR in our sample.

\begin{tabular}{cccccc}
\hline \hline$r$ & $p$ & $a$ & $b$ & $\sigma$ & $\delta$ \\
\hline \multicolumn{6}{c}{ Clouds } \\
\hline 0.73 & 0.004 & $37.31_{-1.92}^{+1.95}$ & $1.02_{-0.47}^{+0.49}$ & $0.55_{-0.15}^{+0.25}$ & 0.41 \\
\hline \multicolumn{6}{c}{ Galaxies } \\
\hline 0.996 & $\sim 10^{-6}$ & $38.29_{-0.10}^{+0.09}$ & $1.28_{-0.07}^{+0.07}$ & $0.21_{-0.06}^{+0.11}$ & 0.15 \\
\hline
\end{tabular}

Notes. $r$ is the Pearson correlation coefficient, and $p$ is the chance probability. We model the two-parameter correlation using the form $\log L_{\gamma}=a+b \log (\mathrm{SFR}) . \sigma$ is introduced to accommodate intrinsic scatter and measurement errors. $\delta$ is the dispersion of a regression model with standard deviation of $L_{\gamma}^{r}$ from $L_{\gamma}$, where $r$ marks the $L_{\gamma}$ value derived from the regression model. The coefficients of $a, b$, and $\sigma$ are constrained simultaneously by maximizing the joint likelihood function.

though there is some difference in the correlation coefficients and the dispersion of the fit. Particularly, stronger dependences of $L_{1-500 \mathrm{GeV}}$ on gas mass $M$ for molecular clouds and $L_{1-500 \mathrm{GeV}}$ on SFR for star-forming galaxies are indicated by the Pearson correlation coefficient $r>0.9$ and the chance probability $p<10^{-4}$. Figure 2 shows the correlation between gamma-ray luminosity and mass $M$ of the molecular cloud sample.

As described above, the gamma-ray emission from starforming regions originates from $\mathrm{CR}$ interactions. In principle, $L_{\gamma}$ may depend not only on the number of CR sources denoted 

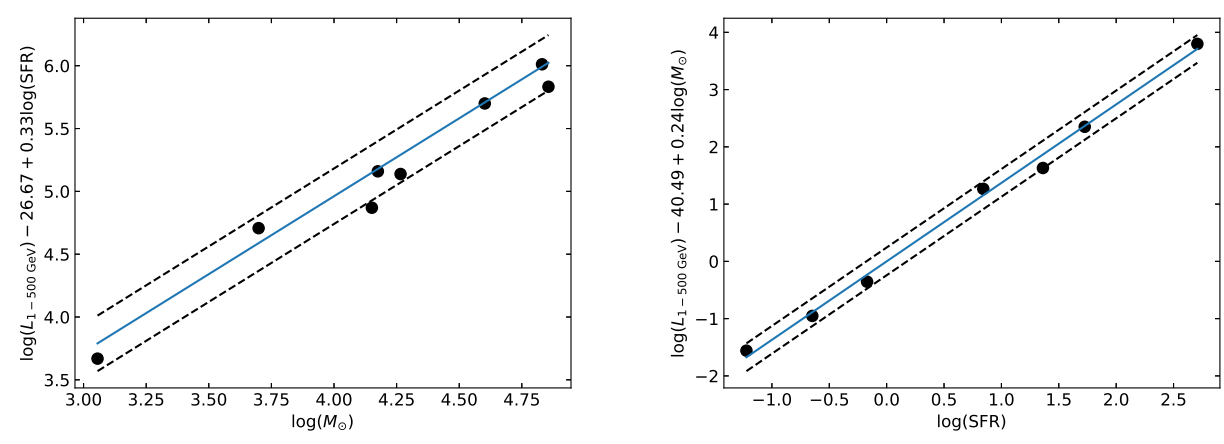

Fig. 3. Three-parameter correlation among $L_{1-500 \mathrm{GeV}}, \mathrm{SFR}$, and $M$ for molecular clouds (left panel) and star-forming galaxies (right panel). The best-fit lines together with their $1 \sigma$ dispersion regions are shown with solid and dashed lines, respectively.
Table 6. Results of the two-parameter correlation for $L_{1-500 \mathrm{GeV}}$ and $M$ in our sample.

\begin{tabular}{cccccc}
\hline \hline$r$ & $p$ & $a$ & $b$ & $\sigma$ & $\delta$ \\
\hline \multicolumn{6}{c}{ Clouds-dense } \\
\hline 0.85 & 0.007 & $30.23_{-0.90}^{+0.89}$ & $0.92_{-0.28}^{+0.28}$ & $0.42_{-0.11}^{+0.18}$ & 0.31 \\
\hline \multicolumn{6}{c}{ Clouds-total } \\
\hline 0.97 & $\sim 10^{-4}$ & $28.90_{-0.55}^{+0.54}$ & $1.02_{-0.14}^{+0.14}$ & $0.19_{-0.06}^{+0.09}$ & 0.15 \\
\hline \multicolumn{7}{c}{ Galaxies } \\
\hline 0.80 & 0.03 & $18.86_{-8.37}^{+8.59}$ & $2.14_{-0.90}^{+0.88}$ & $1.43_{-0.41}^{+0.77}$ & 1.00 \\
\hline
\end{tabular}

Notes. $r$ is the Pearson correlation coefficient, and $p$ is the chance probability. We model the two-parameter correlation using the form $\log L_{\gamma}=a+b \log (M) . \sigma$ is introduced to accommodate intrinsic scatter and measurement errors. $\delta$ is the dispersion of a regression model with standard deviation of $L_{\gamma}^{r}$ from $L_{\gamma}$, where $r$ marks the $L_{\gamma}$ value derived from the regression model. The coefficients of $a, b$, and $\sigma$ are constrained simultaneously by maximizing the joint likelihood function.

by the SFR, but also on the mass of the target gas that is denoted by $M$. Therefore, it is interesting to investigate the possible multiparameter correlation among $L_{\gamma}$, SFR, and $M$. The likelihood function can also be conveniently applied to the three-parameter correlation case by introducing an additional term of $c \times y_{i}$, that is, $\log L_{\gamma}=a+b \log (\mathrm{SFR})+c \log (M)$. The relative dependence of $L_{\gamma}$ on SFR or $M$ is shown clearly through this three-parameter correlation analysis, which is helpful to reveal the mechanism of gamma-ray emission.

The results of three-parameter correlations among $L_{\gamma}$, SFR, and $M$ for molecular clouds and star-forming galaxies are reported in Table 7 . The best-fit correlations are

$$
\begin{aligned}
& \log \left(L_{1-500 \mathrm{GeV}}\right)=\left(26.67_{-1.90}^{+2.02}\right)+\left(-0.33_{-0.29}^{+0.29}\right) \log (\mathrm{SFR}) \\
& +\left(1.23_{-0.22}^{+0.22}\right) \log (M)
\end{aligned}
$$

for molecular clouds and

$$
\begin{aligned}
& \log \left(L_{1-500 \mathrm{GeV}}\right)=\left(40.49_{-2.25}^{+2.24}\right)+\left(1.37_{-0.12}^{+0.12}\right) \log (\mathrm{SFR}) \\
&+\left(-0.24_{-0.25}^{+0.25}\right) \log (M)
\end{aligned}
$$

for star-forming galaxies, respectively. The derived $1 \sigma$ errors of these coefficients are listed in Table 7. The Pearson correlation coefficient $r>0.9$ and chance probability $p<10^{-4}$ suggest strong correlations among $L_{1-500 \mathrm{GeV}}$, SFR, and $M$. For molecular clouds, the weak dependence on SFR, as indicated by $b \sim 0$ within the error box, shows that the $1-500 \mathrm{GeV}$ gamma-ray
Table 7. Results of the three-parameter correlation for $L_{1-500 \mathrm{GeV}}$, SFR, and $M$ in our sample.

\begin{tabular}{ccccccc}
\hline \hline$r$ & $p$ & $a$ & $b$ & $c$ & $\sigma$ & $\delta$ \\
\hline \multicolumn{7}{c}{ Clouds } \\
\hline 0.979 & $\sim 10^{-5}$ & $26.67_{-1.90}^{+2.02}$ & $-0.33_{-0.29}^{+0.29}$ & $1.23_{-0.22}^{+0.22}$ & $0.19_{-0.06}^{+0.10}$ & 0.12 \\
\hline \multicolumn{7}{c}{ Galaxies } \\
\hline 0.997 & $\sim 10^{-6}$ & $40.49_{-2.25}^{+2.24}$ & $1.37_{-0.12}^{+0.12}$ & $-0.24_{-0.25}^{+0.25}$ & $0.21_{-0.07}^{+0.14}$ & 0.12 \\
\hline
\end{tabular}

Notes. $r$ is the Pearson correlation coefficient, and $p$ is the chance probability. We model the three-parameter correlation using the form $\log L_{\gamma}=a+b \log (\mathrm{SFR})+c \log (M) . \sigma$ is introduced to accommodate intrinsic scatter and measurement errors. $\delta$ is the dispersion of a regression model with standard deviation of $L_{\gamma}^{\mathrm{r}}$ from $L_{\gamma}$, where $r$ marks the $L_{\gamma}$ value derived from the regression model. The coefficients of $a, b, c$, and $\sigma$ are constrained simultaneously by maximizing the joint likelihood function.

luminosity is principally proportional to the gas mass $M$. However, the results for galaxies are the opposite. $c=-0.24_{-025}^{+0.25}$ means that the gamma-ray luminosity of galaxies depends little on the total gas mass $M$. There is a clear trend that $L_{1-500 \mathrm{GeV}}$ increases with SFR. These results are consistent with the twoparameter correlations analysis. The dispersion and linear coefficient for the three-parameter correlation are almost the same as those of the two-parameter fit of $L_{1-500 \mathrm{GeV}}-M$ for molecular clouds and $L_{1-500 \mathrm{GeV}}-\mathrm{SFR}$ for star-forming galaxies, respectively. Introducing the third parameter into the three-parameter correlation does not improves the fit significantly, which implies a weak dependence of $L_{\gamma}$ on SFR for molecular clouds, and on $M$ for star-forming galaxies. The regression lines together with $1 \sigma$ dispersion regions are also presented in Fig. 3. For a comparison with previous works, we also performed a multi-parameter correlation analysis for molecular clouds and star-forming galaxies using a $0.1-100 \mathrm{GeV}$ gamma-ray luminosity. The results are reported in Table 8

\section{Discussion}

The correlation among $L_{\gamma}, \mathrm{SFR}$, and $M$ is of theoretical interest in understanding the mechanism of $\mathrm{GeV}$ emission in molecular clouds and star-forming galaxies. The formula for estimating the gamma-ray emission quantitatively (Aharonian et al. 2018) reads

$$
\frac{L_{\gamma}\left(\geqslant E_{\gamma}\right)}{10^{34} \mathrm{erg} \mathrm{s}^{-1}}=5.6\left(\frac{M}{10^{5} M_{\odot}}\right)\left(\frac{\eta}{1.5}\right)\left(\frac{u_{\mathrm{CR}}\left(\geqslant 10 E_{\gamma}\right)}{1 \mathrm{ev} \mathrm{cm}^{-3}}\right),
$$


Table 8. Results of the three-parameter correlation for $L_{0.1-100 \mathrm{GeV}}$, SFR, and $M$ in our sample.

\begin{tabular}{ccccccc}
\hline \hline$r$ & $p$ & $a$ & $b$ & $c$ & $\sigma$ & $\delta$ \\
\hline \multicolumn{7}{c}{ Clouds } \\
\hline 0.976 & $\sim 10^{-5}$ & $27.16_{-2.13}^{+1.98}$ & $-0.32_{-0.32}^{+0.30}$ & $1.22_{-0.23}^{+0.24}$ & $0.20_{-0.06}^{+0.10}$ & 0.13 \\
\hline \multicolumn{7}{c}{ Galaxies } \\
\hline 0.998 & $\sim 10^{-8}$ & $35.70_{-1.59}^{+1.61}$ & $1.16_{-0.09}^{+0.09}$ & $0.31_{-0.18}^{+0.18}$ & $0.16_{-0.05}^{+0.09}$ & 0.11 \\
\hline
\end{tabular}

Notes. $r$ is the Pearson correlation coefficient, and $p$ is the chance probability. We model the three-parameter correlation using the form $\log L_{\gamma}=a+b \log (\mathrm{SFR})+c \log (M) . \sigma$ is introduced to accommodate intrinsic scatter and measurement errors. $\delta$ is the dispersion of a regression model with standard deviation of $L_{\gamma}^{\mathrm{r}}$ from $L_{\gamma}$, where $r$ marks the $L_{\gamma}$ value derived from the regression model. The coefficients of $a, b, c$, and $\sigma$ are constrained simultaneously by maximizing the joint likelihood function.

where $M$ is the mass of the relevant region, $\eta$ accounts for the presence of nuclei higher in mass than hydrogen in CRs and interstellar matter, and $u_{\mathrm{CR}}$ is the CR density.

If the molecular clouds in our sample are an active source of CR acceleration, similar to star-forming galaxies, or if the embedded YSOs can contribute CRs at a comparable level of the Galactic CR sea, gamma-ray emission should also show a correlation with CR density (denoted as SFR), smoothly connecting the $L_{\gamma}-$ SFR relationship of star-forming galaxies. Correspondingly, the dependence of $L_{\gamma}$ on the SFR in three-parameters correlation should be much stronger. However, this is not supported by our data analysis. The data set of molecular clouds and star-forming galaxies is located in different regions in the diagram of gamma-ray emissivity $\left(L_{\gamma} / M\right)$ and SFR per unit mass (Fig. 1). The gamma-ray emissivity $\left(L_{\gamma} / M\right)$ remains to be a constant for different SFR/M for Galactic molecular clouds. This is consistent with the gamma-ray's being produced outside of the cloud and with the recent study of diffuse Fermi gamma-ray emission, which seems to trace the total molecular gas content on a global scale (Remy et al. 2018). The nice linear correlation between $L_{\gamma}$ and total mass for molecular clouds is demonstrated by the slope of $1.02_{-0.14}^{+0.14}$. In other words, the CRs that produce the dominant part of the gamma-ray emission in molecular clouds may be accelerated outside, as expected from the passive-cloud scenario. These molecular clouds float in the sea of the Galactic $\mathrm{CRs}$, and the produced gamma-ray emission is proportional to the total gas mass under the hypothesis that the non-violent change CRs flux penetrates the clouds. The embedded massive stars may contribute to CRs secondarily or account for some CR hot spots around clusters of young stellar objects (e.g., Marchili et al. 2018). The Pearson correlation coefficient of $L_{\gamma}-$ SFR for molecular clouds is also good from a statistical point of view, but it may simply reflect the $L_{\gamma}-M_{\text {dense }}$ correlation, as $M_{\text {dense }}$ is a proxy of SFR. It could be naturally explained by the combination of an only mildly varying fraction of dense gas mass (see Table 1) and a tight $L_{\gamma}-M$ correlation.

For star-forming galaxies, the Pearson correlation coefficient and dispersion of $L_{\gamma}-M$ correlation demonstrates that the twoparameter relationship is poor, which is consistent with results of the three-parameter correlation. The gamma-ray luminosity of star-forming galaxies can be parameterized by $L_{\gamma} \sim f_{\text {cal }} L_{\mathrm{CR}}$, where $L_{\mathrm{CR}}$ is the CR luminosity in galaxies, which is proportional to the SFR, and $f_{\text {cal }}$ is the calorimetric factor denoting the fraction of the energy of CRs converted into secondary pions. Star-forming galaxies with an SFR $>10 M_{\odot} \mathrm{yr}^{-1}$ are close to the calorimetric limit (e.g., Lacki et al. 2011; Wang \& Fields 2018 ), that is, $f_{\text {cal }} \simeq 1$. If the CR calorimetry hypothesis were to hold, the slope of the relation between the gamma-ray luminosity and SFR would be unity. The observed slope of the relation is steeper than unity, however (Abdo et al. 2010a; Ackermann et al. 2012a; Tang et al. 2014; Peng et al. 2016), indicating that galaxies with lower SFRs may have smaller $f_{\text {cal }}$. Pfrommer et al. (2017) reproduced the observed relation between far-infrared and gamma-ray emission using magnetohydrodynamical galaxy formation simulations with self-consistent CR physics. They found that the calorimetric factor $f_{\text {cal }}$ decreases smoothly toward lower SFRs due to the increasing adiabatic losses of CRs.

\section{Conclusions}

We have analyzed the Fermi-LAT data of eight Galactic molecular clouds in the Gould Belt. Through a comparative study of the correlations among the gamma-ray luminosity, SFR, and gas mass $M$, we found that the gamma-ray luminosity of molecular clouds is strongly dependent on the total gas mass $M$ and weakly dependent on SFR. The SFR inside molecular clouds makes minor contribution to the gamma-ray emission. The results of star-forming galaxies are just the opposite. A tight dependence between the gamma-ray luminosity and SFR is found, with little dependence on $M$. The different empirical correlations found in molecular clouds and star-forming galaxies indicates that different mechanisms produce the gamma-ray emission, with Galactic clouds being more of a passive target to interact with CRs. The gamma-ray emission in molecular clouds originates predominantly from the interaction of diffuse galactic CRs. Star-forming galaxies are effective reservoirs for CRs, and a significant fraction of CR energy is transferred into secondary gamma-rays, at least for those with $\mathrm{GeV}$ emission that are observed by Fermi-LAT.

Acknowledgments. This work is partially supported by the National Key R \& D program of China under the grant 2018YFA0404203 and 2017YFA0402600, and the NSFC grants 11625312, 11851304, U1731238, 11565010, and 11725313. F.K.P acknowledges support from the Doctoral Starting up Foundation of Guizhou Normal University 2017 (GZNUD[2017] 33) and the open project of Guangxi Key Laboratory for Relativistic Astrophysics. Q.J.Z. acknowledges support from the science and technology innovation talent team (grant (2015)0415), the High Level Creative Talents (grant (2016)-4008) and Innovation Team Foundation of the Education Department (grant [2014]35) of Guizhou Province. D.L. acknowledges support from the International Partnership Program of Chinese Academy of Sciences, Grant No.114A11KYSB20160008 and the CAS Strategic Priority Research Program No. XDB23000000. We thank R.Z. Yang, R.-Y. Liu, J.Z. Wang, K.P. Qiu and H. Chen for useful discussions. This work has made use of data and software provided by the Fermi Science Support Center.

\section{References}

Abdo, A. A., Ackermann, M., Ajello, M., et al. 2009, ApJ, 703, 1249 Abdo, A. A., Ackermann, M., Ajello, M., et al. 2010a, A\&A, 523, L2 Abdo, A. A., Ackermann, M., Ajello, M., et al. 2010b, ApJ, 710, A133 Abdo, A. A., Ajello, M., Allafort, A., et al. 2013, ApJS, 208, 17

Acero, F., Aharonian, F., Akhperjanian, A. G., et al. 2009, Science, 326, 1080

Acero, F., Ackermann, M., Ajello, M., et al. 2015, ApJS, 218, 23

Ackermann, M., Ajello, M., Baldini, L., et al. 2011a, ApJ, 726, 81 Ackermann, M., Ajello, M., Allafort, A., et al. 2011b, Science, 334, 1103 Ackermann, M., Ajello, M., Allafort, A., et al. 2012a, ApJ, 755, 164 Ackermann, M., Ajello, M., Allafort, A., et al. 2012b, ApJ, 755, 22 Ackermann, M., Ajello, M., Allafort, A., et al. 2012c, ApJ, 756, 4 Ackermann, M., Ajello, M., Atwood, W. B., et al. 2012d, ApJ, 750, 3 
Ackermann, M., Ajello, M., Albert, A., et al. 2017, ApJ, 836, 208

Aharonian, F. A. 2001, Space Sci. Rev., 99, 187

Aharonian, F., Yang R., \& de Oña Wilhelmi, E. 2018, ArXiv e-prints [arXiv: 1804.02331$]$

Araudo, A. T., Romero, G. E., Bosch-Ramon, V., \& Paredes, J. M. 2007, A\&A, 476, 1289

Atwood, W. B., Abdo, A. A., Ackermann, M., et al. 2009, ApJ, 697, 1071

Bosch-Ramon, V., Romero, G. E., Araudo, A. T., \& Paredes, J. M. 2010, A\&A 511, A8

Braun, R., Thilker, D. A., Walterbos, R. A. M., \& Corbelli, E. 2009, ApJ, 695, 937

Carpenter, J. M., \& Hodapp, K. W. 2008, Handbook of Star Forming Regions, Volume I (San Francisco: ASP), 4, 899

Casandjian, J.-M. 2012, AIP Conf. Ser., 1505, 37

Case, G. L., \& Bhattacharya, D. 1998, ApJ, 504, 761

Cillis, A. N., Torres, D. F., \& Reimer, O. 2005, ApJ, 621, 139

Dame, T. M., Hartmann, D., \& Thaddeus, P. 2001, ApJ, 547, 792

del Valle, M. V., Romero, G. E., Luque-Escamilla, P. L., Martí, J., \& Ramón Sánchez-Sutil J. 2011, ApJ, 738, 115

Feigelson, E. D., \& Babu, G. J. 1992, ApJ, 397, 55

Förster Schreiber, N. M., Genzel, R., Lutz, D., \& Sternberg, A. 2003, ApJ, 599, 193

Foreman-Mackey, D., Hogg, D. W., Lang, D., \& Goodman, J. 2013, PASP, 125 306

Fukui, Y., Kawamura, A., Minamidani, T., et al. 2008, ApJS, 178, 56

Gabici, S., Aharonian, F. A., \& Blasi, P. 2007, Ap\&SS, 309, 365

Gao, Y., \& Solomon, P. M. 2004, ApJ, 606, 271

Grenier, I. A., Casandjian, J.-M., \& Terrier, R. 2005, Science, 307, 1292

Griffin, R. D., Dai, X., \& Thompson, T. A. 2016, ApJ, 823, L17

Heiderman, A., Evans, N. J., II, Allen, L. E., Huard, T., \& Heyer, M. 2010, ApJ, 723, 1019-37

Hughes, A., Staveley-Smith, L., Kim, S., Wolleben, M., \& Filipović, M. 2007, MNRAS, 382, 543

Isobe, T., Feigelson, E. D., Akritas, M. G., \& Babu, G. J. 1990, ApJ, 364, 104

Knude, J. 2010, ArXiv e-prints [arXiv:1006. 3676]

Lacki, B. C., Thompson, T. A., Quataert, E., Loeb, A., \& Waxman, E. 2011, ApJ, 734, 107

Lada, C. J., Lombardi, M., \& Alves, J. F. 2010, ApJ, 724, 687

Lada, C. J., Forbrich, J., Lombardi, M., \& Alves, J. F. 2012, ApJ, 745, 190

Lenc, E., \& Tingay, S. J. 2006, AJ, 132, 1333

Leroy, A., Bolatto, A., Stanimirovic, S., et al. 2007, ApJ, 658, 1027
Li, H., Li, D., Qian, L., et al. 2015, ApJS, 219, 20

Lombardi, M., Lada, C. J., \& Alves, J. 2008, A\&A, 480, 785

Lombardi, M., Lada, C. J., \& Alves, J. 2010, A\&A, 512, A67

Lombardi, M., Alves, J., \& Lada, C. J. 2011, A\&A, 535, A16

Lorimer, D. R., Faulkner, A. J., Lyne, A. G., et al. 2006, MNRAS, 372, 777

Luhman, K. L. 2008, Handbook of Star Forming Regions, Volume II (San Francisco: ASP), 5, 169

Marchili, N., Piano, G., Cardillo, M., et al. 2018, A\&A, 615, A82

Maurin, G., Marcowith, A., Komin, N., Krayzel, F., \& Lamanna, G. 2016, A\&A, 591, A71

Mizuno, A., Hayakawa, T., Tachihara, K., et al. 1999, PASJ, 51, 859

Neronov, A., Semikoz, D. V., \& Taylor, A. M. 2012, Phys. Rev. Lett., 108, 051105 Neronov, A., Malyshev, D., \& Semikoz, D. V. 2017, A\&A, 606, A22

Nieten, C., Neininger, N., Guélin, M., et al. 2006, A\&A, 453, 459

Paladini, R., Montier, L., Giard, M., et al. 2007, A\&A, 465, 839

Peng, F.-K., Wang, X.-Y., Liu, R.-Y., Tang, Q.-W., \& Wang, J.-F. 2016, ApJ, 821, L20

Pfrommer, C., Pakmor, R., Simpson, C. M., \& Springel, V. 2017, ApJ, 847, L13 Pokhrel, R., Gutermuth, R., Ali, B., et al. 2016, MNRAS, 461, 22

Remy, Q., Grenier, I. A., Marshall, D. J., \& Casandjian, J. M. 2018, A\&A, 611, A51

Shen, Z.-Q., Liang, Y.-F., Duan, K.-K., et al. 2018, ArXiv e-prints [arXiv: 1801.06075]

Stanimirovic, S., Staveley-Smith, L., Dickey, J. M., Sault, R. J., \& Snowden, S. L. 1999, MNRAS, 302, 417

Staveley-Smith, L., Kim, S., Calabretta, M. R., Haynes, R. F., \& Kesteven, M. J. 2003, MNRAS, 339, 87

Strong, A. W., Porter, T. A., Digel, S. W., et al. 2010, ApJ, 722, L58

Tang, Q.-W., Wang, X.-Y., \& Tam, P.-H. T. 2014, ApJ, 794, 26

Vázquez-Semadeni, E., Banerjee, R., Gómez, G. C., et al. 2011, MNRAS, 414, 2511

VERITAS Collaboration (Acciari, V. A., et al.) 2009, Nature, 462, 770

Vladimirov, A. E., Digel, S. W., Jóhannesson, G., et al. 2011, Comput. Phys. Commun., 182, 1156

Wang, X., \& Fields, B. D. 2018, MNRAS, 474, 4073

Wilke, K., Klaas, U., Lemke, D., et al. 2004, A\&A, 414, 69

Wu, J., Evans, N. J., II, Gao, Y., et al. 2005, ApJ, 635, L173

Yang, R.-Z., \& Aharonian, F. 2017, A\&A, 600, A107

Yang, R.-z., de Oña Wilhelmi, E., \& Aharonian, F. 2014, A\&A, 566, A142

Yang, R.-z., de Oña Wilhelmi, E., \& Aharonian, F. 2018, A\&A, 611, A77

Yin, J., Hou, J. L., Prantzos, N., et al. 2009, A\&A, 505, 497 